Carnets de géographes

GÉOGRAPHES.

\title{
Conflits environnementaux en territoire industriel
}

Réappropriation territoriale et émergence d'une justice environnementale. Le cas de l'étang de Fos-sur-Mer

\section{Clara Osadtchy}

\section{(2) OpenEdition}

\section{Journals}

Édition électronique

URL : http://journals.openedition.org/cdg/332

DOI : $10.4000 /$ cdg.332

ISSN : 2107-7266

Éditeur

UMR 245 - CESSMA

\section{Référence électronique}

Clara Osadtchy, "Conflits environnementaux en territoire industriel », Carnets de géographes [En ligne], 8 | 2015, mis en ligne le 01 septembre 2015, consulté le 24 septembre 2020. URL : http:// journals.openedition.org/cdg/332 ; DOI : https://doi.org/10.4000/cdg.332

Ce document a été généré automatiquement le 24 septembre 2020.

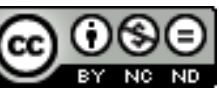

La revue Carnets de géographes est mise à disposition selon les termes de la Licence Creative Commons Attribution - Pas d'Utilisation Commerciale - Pas de Modification 4.0 International. 


\title{
Conflits environnementaux en territoire industriel
}

\author{
Réappropriation territoriale et émergence d'une justice
} environnementale. Le cas de l'étang de Fos-sur-Mer

\section{Clara Osadtchy}

1 Il n'est pas un jour sans que ne soit médiatisé un nouveau conflit lié à l'exploitation des ressources naturelles, à l'aménagement du territoire ou à des problèmes sanitaires et de pollution. Ces conflits font référence, à la fois à la multiplication de la conflictualité dans les territoires, à l'intégration sociale des préoccupations environnementales et contribuent à la popularisation de la notion de justice environnementale qui s'exprime comme un nouveau mode revendicatif basé sur la dénonciation d'injustices environnementales et sociales, ethniquement marquées ou non. Ces dernières années, les conflits territoriaux portant sur l'aménagement et l'environnement ont été particulièrement médiatisés en France, en particulier ceux liés à l'opposition à la construction de l'aéroport de Notre-Dame-des-Landes et au barrage de Sivens. L'aménagement du territoire est devenu un enjeu politique majeur du fait des difficultés des projets à voir le jour face à des dynamiques d'opposition locale diverses.

2 Cette recherche a pour objectif d'étudier les significations socio-spatiales de plusieurs dynamiques conflictuelles relatives à l'aménagement et à l'environnement en territoire fortement industrialisé. L'analyse porte sur les raisons et la genèse de mobilisations d'habitants et d'acteurs à l'œuvre depuis les années 2000 autour de l'étang de Berre et du golfe de Fos (Bouches-du-Rhône), territoire en partie annexé pour les besoins du port autonome de Marseille depuis un siècle. Les conflits sont ici vus comme des analyseurs sociaux, révélateurs des territoires, des sociétés qui les composent et des tensions qui traversent leurs relations. Leur analyse se décline en l'étude de l'émergence des antagonismes (historique, représentations sociales en jeu), des formes d'expression des oppositions (registres discursifs et stratégiques), des dimensions spatiales et multiscalaires des mobilisations (inscription territoriale, champs d'action, alliances) et des modes de régulation (négociation, concertation, arbitrages, etc.) dans le temps et dans l'espace. 
3 Cette thèse contribue à la compréhension de l'avènement d'une conflictualité dans l'aménagement du territoire, résultat d'un contexte national de «crise». Pourquoi et comment des conflits portant sur l'aménagement et l'environnement peuvent-ils prendre forme en territoire industriel ? Quels sont les mécanismes de concernement et de mobilisation des habitants et les dynamiques favorisant l'entrée en conflit? Quels arguments et quelles stratégies mettent-elles en œuvre pour intégrer l'espace public ? Les conflits peuvent-ils mettre en évidence des assymétries, des inégalités, des phénomènes territoriaux qui s'expriment sur les plans sociaux-spatiaux au travers de ces conflits? Comment s'articule l'intervention de politiques de démocratie environnementale et quelle en est la portée?

La méthode employée dans cette recherche est qualitative et procède d'une démarche inductive et constructiviste. Elle s'appuie sur des sources et données de nature diverse : corpus de plus de 300 documents (période allant de 1994 à 2014); revue de presse régionale et locale ; 41 entretiens semi-directifs; observation non participante à des évènements (2010 à 2012).

5 La première partie de cette thèse montre que l'approche de ces conflits par la notion de risque apparait comme un cadre d'analyse pertinent pour aborder l'étude des conflits portant sur l'environnement industriel. Ces conflits sont en effet caractérisés par l'existence territoriale de risques industriels qui sont à la fois matérialité et construits sociaux et sont constitutifs des spécificités des territoires industriels: existence d'inégalités environnementales, processus de disqualification sociale et environnementale, construction sociale des risques, politiques de gestion des risques et action publique de sécurité industrielle tournée vers la maîtrise de l'urbanisation, existence d'arènes de débat institutionnalisées ou sauvages.

6 La seconde partie met en évidence l'influence majeure de l'industrie sur l'identité du territoire de l'étang de Berre et du golfe de Fos. Depuis le début du $20^{\mathrm{e}}$ siècle, le territoire s'est en effet développé pour les besoins du port de Marseille; le développement industriel a dès lors largement façonné le paysage, l'aménagement et l'urbanisme. L'étude diachronique de la place prise par la notion d'environnement permet de situer la construction socio-politique des problèmes d'environnement à partir des années 1960, intrinsèquement liée à l'industrialisation de l'étang de Berre puis du golfe de Fos. En nommant les externalités négatives du développement industriel, des coalitions d'acteurs locaux ont forgé leur propre définition du terme de pollution. Ces mobilisations ont permis de fédérer des habitants et des acteurs locaux dans un même mouvement de dénonciation et de revendication. Il est apparu qu'à l'apogée de cette contestation ont succédé, à partir de 1972, des réformes publiques successives reconnaissant la nécessité d'une régulation de l'activité industrielle.

7 La troisième partie de cette recherche propose une visite guidée de la conflictualité née sur le golfe de Fos, puis à La Mède entre les années 2000 et 2010, autour des projets d'implantation d'un incinérateur, de plusieurs terminaux gaziers et de la mise en œuvre d'un plan d'urbanisme (le plan de prévention des risques technologiques d'une raffinerie). Nous distinguons et analysons différentes notions qui s'entendent comme des séquences dans l'étude de ces conflits : le concernement (activation des publics), la mobilisation (objet, structuration, registres discursifs) et le conflit (dynamique relationnelle entre acteurs, trajectoire des projets contestés). Nous mettons en évidence la portée institutionnelle de ces conflits (ouverture au public, amélioration environnementale, intégration territoriale des projets portuaires). Il apparaît que ces 
mobilisations d'habitants et d'élus sont des espaces de légitimation locale et de réappropriation territoriale, et que les mécanismes de montée en généralité observés participent à la reformulation de l'intérêt général.

Figure 1 Situation géographique de l'étang de Berre et du golfe de Fos-sur-Mer.
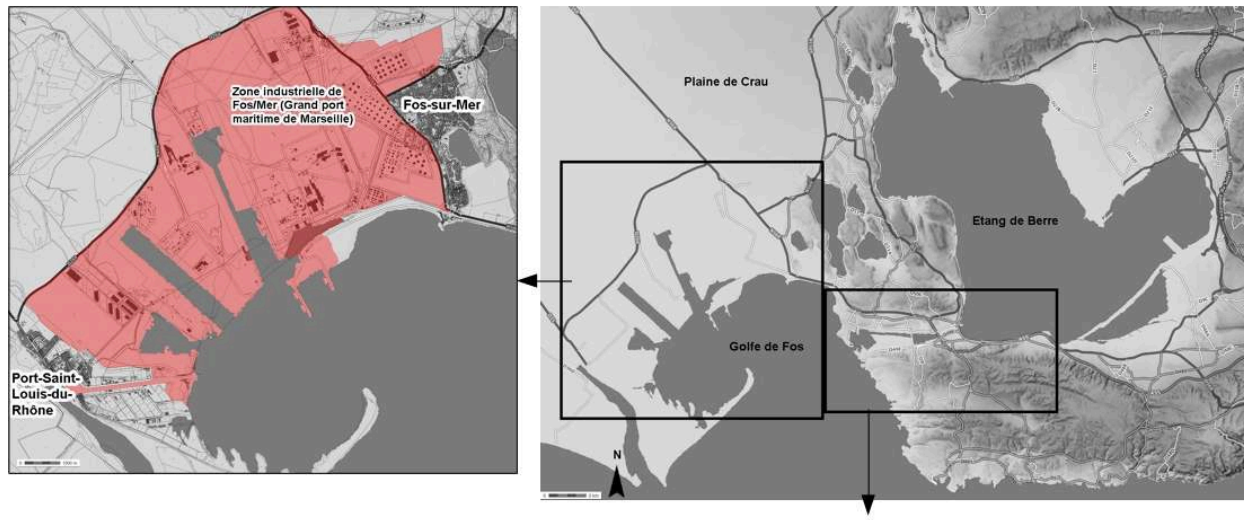

Figure : carte de situation, des espaces urbains et industriels contigus (carte: C.Osadtchy)

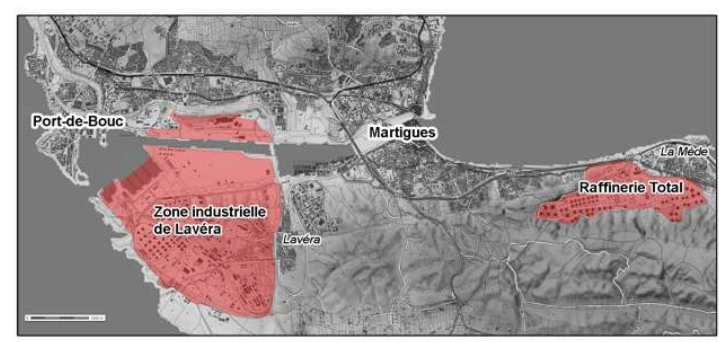

Figure 2 Un territoire industrialo-portuaire aux usages multiples. Sport de mer, quartier urbain et zone industrielle de Lavéra (Martigues).

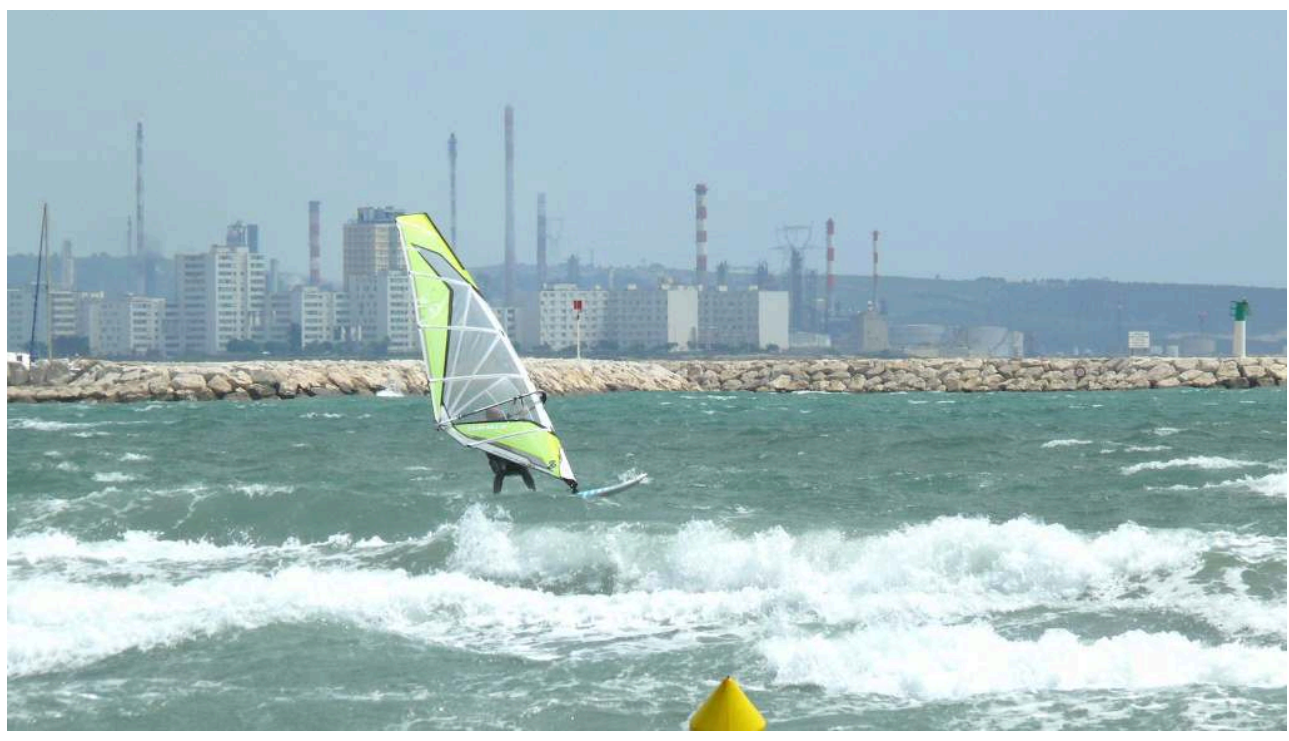

\section{Discipline}

Géographie régionale et aménagement de l'espace et urbanisme 


\section{Directeur}

Cyria EMELIANOFF, Professeur, Université du Maine - Le Mans

Jacques CHEVALIER, Professeur Emérite, Université du Maine - Le Mans

\section{Université}

Université du Maine (Le Mans)

Membres du jury de thèse, soutenue le 6 juillet 2015

Guy BAUDELLE, Professeur, Université de Rennes, rapporteur,

Philippe SUBRA, Professeur, Université Paris 8, rapporteur,

Emmanuel MARTINAIS, Chargé de recherche, École nationale des travaux publics

de l'État, examinateur,

Patrice MELÉ, Professeur, Université François Rabelais, examinateur

Situation professionnelle à l'issue de la thèse

Chercheure-associée au laboratoire ESO Le Mans

\section{Courriel de l'auteur}

claraosad[at]yahoo.fr

INDEX

Thèmes : Carnets de soutenances 